$>$ Le haut degré de conservation des voies de signalisation impliquées dans la régulation de la longévité justifie l'utilisation d'organismes aussi variés que les nématodes, les souris, ou encore les lémuriens pour l'étude du vieillissement humain. La complémentarité de ces modèles permet d'aborder différentes questions: celles des interactions entre les facteurs génétiques et environnementau, de la conservation des fonctions auditives, de la lutte contre la sénescence des cellules souches neurales ou encore de l'exploration des aptitudes cérébrales depuis l'expression génique jusqu'aux comportements cognitifs et sociaux. Tout en différenciant les aspects physiologiques et pathologiques des processus liés à l'âge, nous soulignerons l'intérêt des technologies de pointe pour une meilleure compréhension des mécanismes régissant le vieillissement. <

Le vieillissement est un processus complexe et encore mal connu. Le recours aux modèles animaux est indispensable pour étudier les mécanismes physiologiques $\mathrm{du}$ vieillissement et les mécanismes pathologiques associés. II n'existe pas de modèle unique parfait mais chaque modèle a son utilité. Dans cet esprit, nous présentons quatre modèles différents, ayant chacun une pertinence dans un des aspects de l'étude du vieillissement: Caenorhabditis elegans facilite l'étude de gènes impliqués dans la longévité (partie rédigée par $S$. Galas, M.-T. Château et P. Pomiès) ; la souris constitue un modèle intéressant pour l'étude du vieillissement sensoriel auditif et de la presbyacousie (J. Wang, J. Menardo et J.-L. Puel) ; les rongeurs représentent également un modèle de choix pour explorer comment les cellules souches neurales du cerveau se comportent lors du vieillissement (J.-P. Hugnot); enfin, l'étude du vieillissement cérébral et des maladies neurodégénératives associées nécessite une approche prenant en compte l'évolution du cerveau d'où la pertinence de l'utilisation d'un petit primate lémurien, le microcèbe murin Microcebus murinus (J.-M. Verdier et G. Devau).

\section{Aperçu de la diversité des modèles animaux dédiés à l'étude du vieillissement}
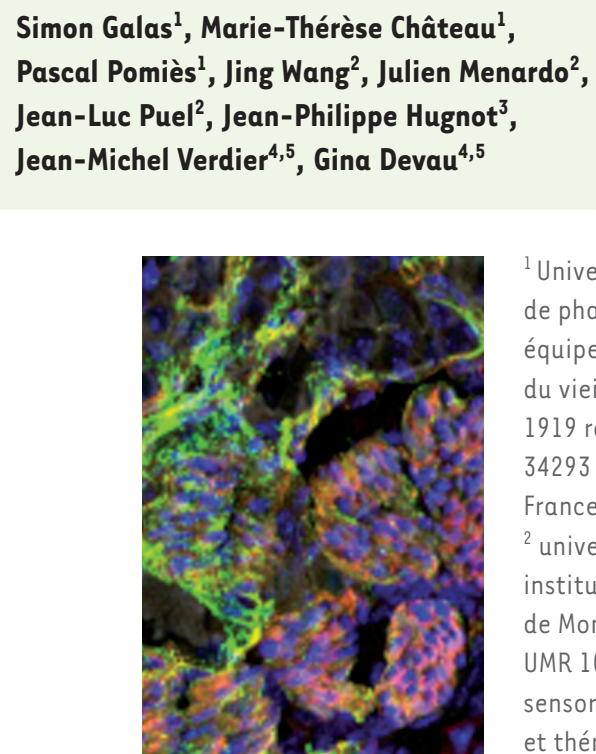

${ }^{1}$ Université Montpellier 1, faculté de pharmacie, CNRS UMR 5237, équipe biotechnologies du vieillissement, 1919 route de Mende, 34293 Montpellier Cedex 5, France ;

${ }^{2}$ université Montpellier l, institut des neurosciences de Montpellier (INM)-Inserm UMR 1051, équipe pathologies sensorielles, neuroplasticité et thérapies, 80 rue Augustin Fliche, 34295 Montpellier, France ;

${ }^{3}$ université Montpellier 2 ; INM-Inserm UMR 1051, équipe plasticité cérébrale, cellules souches et tumeurs gliales, 80 rue Augustin Fliche, 34295 Montpellier, France; ${ }^{4}$ unité mixte mécanismes moléculaires dans les démences neurodégénératives, université Montpellier 2, Inserm U710, 34095 Montpellier, France; 5 École pratique des hautes études, 34095 Montpellier, France.

simon.galas@univ-montpl.fr Nobel de chimie (2008) pour la

Naître, vieillir et mourir avec 959 cellules : les enseignements Difficile de classer dans une même catégorie les découvertes issues du nématode Caenorhabditis elegans. Ce petit ver (long de $1 \mathrm{~mm}$ ) a déjà été distingué par deux prix Nobel de médecine $(2002,2006)$ pour ses contributions aux découvertes de l'apoptose et du mécanisme d'ARN découverte et l'utilisation de la protéine fluorescente verte, la GFP (green fluorescent protein).

Qu'en est-il de C. elegans et du vieillissement? Depuis une trentaine d'années, l'intérêt de ce modèle réside dans trois caractéristiques majeures : la connaissance de son génome dès 1998 [1] ; une durée de vie moyenne de 17 jours, facilitant les études sur la génétique de la longévité ; une simplicité d'organisation par rapport aux

Cette synthèse rapporte des travaux effectués dans les laboratoires de Montpellier qui traitent, sous divers aspects fonctionnels, de questions relatives au vieillissement. Les modèles présentés et sélectionnés sont ceux qui sont actuellement en cours d'étude dans les laboratoires Montpellierains fédérés au sein du Montpellier Aging Network (http://www.inmfrance.com/M.A.N/). 


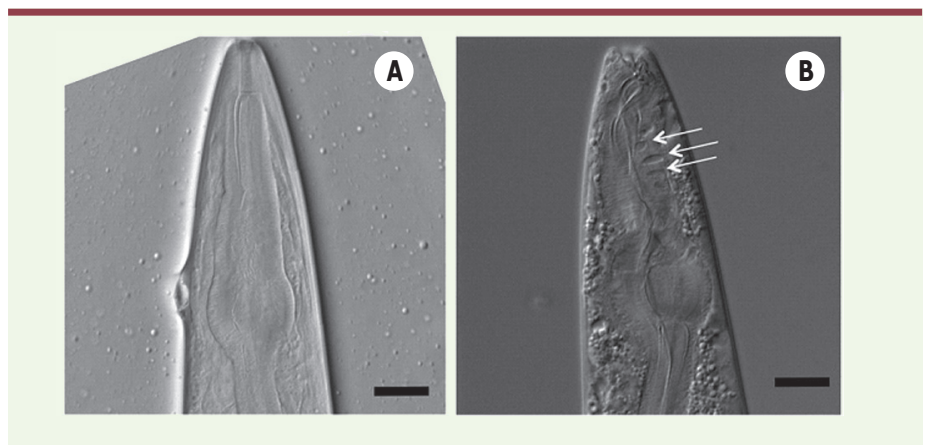

Figure 1. Observation, en microscopie nomarski, de la tête d'un nématode (Caenorhabditis elegans). A. Jeune adulte. B. Adulte âgé de 7 jours. La présence de désorganisations (flèches blanches) avec apparition de cavités au sein du tissu musculaire est caractéristique du processus de vieillissement. Barre $=0,01 \mathrm{~mm}$.

organismes plus complexes, puisqu'au stade adulte ce ver n'est formé que de 959 cellules.

Dès 1982, l'utilisation d'hybrides de vers de souches françaises et anglaises a permis à Johnson et Wood [2] d'estimer à entre 20 et 50 \% la part de l'influence des facteurs héréditaires dans le déterminisme du vieillissement. Des études ultérieures ont montré qu'il était possible de doubler la durée de vie du ver en diminuant l'activité du gène Daf-2 [3], un homologue du gène humain codant pour le récepteur de I'IGF-1 (Insulin like growth factor-1). Ainsi, le vieillissement du ver impliquerait un programme génétique doté d'une certaine élasticité puisque modulable expérimentalement. Daf-2 est le chef de file d'un groupe de gènes appelés « gérontogènes » ou « modulateurs du vieillissement ». Des actions simultanées sur certains de ces gènes ont permis de multiplier jusqu'à six fois la longévité du ver, suggérant que ces «gérontogènes » pourraient fonctionner de manière indépendante. Toutefois, une étude récente [4] révèle la complexité du mode d'action de ces gènes du vieillissement. Ces expériences ont ainsi pu démontrer que le gain de longévité observé chez les vers mutants Daf-2 résulterait de la somme des influences positives et négatives de plusieurs gènes et de leurs produits (protéines) sur le vieillissement. En résumé, des réseaux de gènes aux effets parfois contraires et se régulant entre eux produiraient, in fine, un effet sur la longévité.

Outre qu'il a permis de préciser l'organisation fonctionnelle des « gérontogènes », le ver permet aussi de tester d'anciennes théories du vieillissement. Dès 1956, Harman a proposé sa théorie radicalaire ou encore théorie du stress oxydatif du vieillissement. En 1972, ce même auteur précise que le vieillissement résulte d'un déclin fonctionnel induit par une accumulation de dommages moléculaires et cellulaires telle qu'elle sature les capacités d'autoréparation de l'organisme (un exemple de lésion tissulaire caractéristique est donné dans la Figure 1). Classée parmi les théories gérontologistes du vieillissement - qui tentent d'expliquer le « comment »du vieillissement à l'inverse des théories évolutionnistes qui en recherchent l'« origine »-, la théorie radicalaire va inciter les chercheurs à établir un lien de causalité entre le vieillissement et son induction par les radicaux libres. Cependant, des travaux récents du laboratoire de Hekimi viennent d'apporter un nouvel éclairage [5]. L'observation d'une augmentation de longévité consécutive à une diminution des niveaux d'enzymes responsables de l'élimination de radicaux libres laisse supposer que les effets du stress oxydant sur le ver seraient la conséquence plutôt que la cause du vieillissement $(\rightarrow)$ [51]. Plus récemment, le même laboratoire [6] a pu montrer qu'un traitement par les antioxydants produit un effet négatif sur la longévité !

$(\rightarrow)$ Voir l'article de C. Terret et F. Solari, page 311

Depuis que ces travaux ont débuté,

de ce numéro plusieurs modulateurs de la longévité ont été identifiés chez le ver et répertoriés par certains laboratoires [7]. De telles découvertes favorisent le défrichage de voies de signalisation nouvelles et potentiellement conservées chez l'homme [8]. L'analyse de polymorphismes génétiques, dont certains sont associés à des longévités notables (centenaires) chez I'homme [9] $\rightarrow$ [ [52] commence à livrer un tableau général de leur implication en tant que régula-

$(\rightarrow)$ Voir l'article d'A. Brunet, page 316 de ce numéro teurs de la sénescence. Quant au modèle qu'offre le ver pour le criblage in vivo de gènes impliqués dans la programmation du vieillissement ou encore l'identification de molécules modulatrices [10], il est particulièrement prometteur !

Toutefois, le nématode ne permet pas d'accéder aux fonctions spécialisées acquises au cours de l'évolution, telles que l'audition, une fonction qui a été explorée dans différents modèles murins.

\section{Contribution des modèles murins à la compréhension de la presbyacousie}

Dans les sociétés où l'espérance de vie ne cesse d'augmenter, la surdité liée à l'âge, ou presbyacousie, est un problème de santé publique. En effet, $70 \%$ des personnes de plus de 65 ans ont des problèmes auditifs [11]. La presbyacousie est une pathologie multifactorielle faisant intervenir une combinaison de facteurs individuels et environnementaux. D'un point de vue physiopathologique, Schuknecht et Gacek [12] ont été les premiers à décrire une dégénérescence de l'organe de Corti (presbyacousie sensorielle), du ganglion spiral (presbyacousie neurale) et de la strie vasculaire (presbyacousie striale) en étudiant des os de rochers humains ${ }^{1}$. À côté de ces données humaines, l'exploration de différentes souches de souris présentant un vieillissement cochléaire constitue un outil de recherche précieux pour mieux appréhender les mécanismes de dégénérescence des structures cochléaires (Tableau I) [13]. Ces modèles animaux soulignent aussi l'importance des facteurs génétiques

\footnotetext{
${ }^{1}$ Le rocher forme l'os temporal avec l'écaille et l'os tympanal.
} 


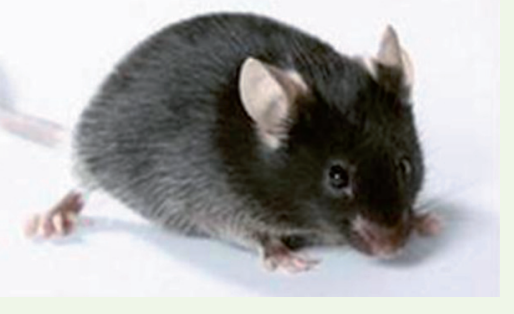

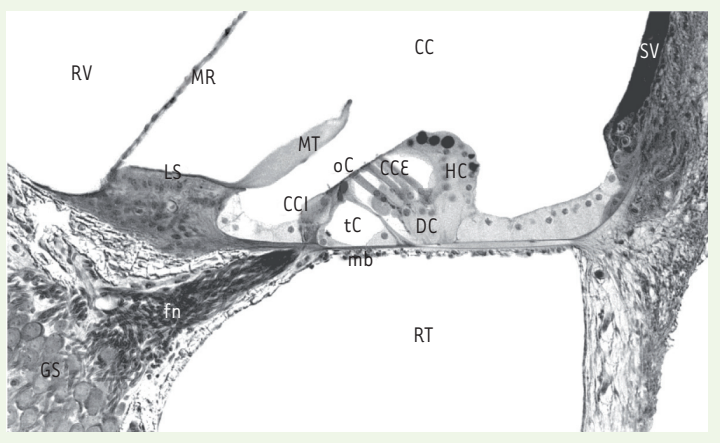

Lignées Localisation

Réf

Fibrocytes du limbe spiral et du ligament spiral

Ahl3

Inconnu

Homozygote

NOD/LtJ

Inconnu

Inconnu

$\begin{array}{ll}\text { Brn4 KO } & \text { Facteur de } \\ \text { transcription lié à l'X }\end{array}$

Hémizygote

129/C57BL/6

Capsule otique

DRASIC KO

Canal mécanorécepteur

Homozygote

C57BL/6/129

Cellules ciliées de l'organe de Corti et cellules du ganglion spiral

PolgA KO

Polymérase ADN mitochondriale

Homozygote

$129 / I C R$

Membrane mitochondriale

$\mathrm{KO}$ Récepteur ACh nicotinique

Homozygote

C57BL/6

Cellules ciliées

$m t-T r$ ARN mitochondrial

Maternelle

A/J

Mitochondrie

Pmca2 ${ }^{d f w} \quad$ ATPase 2

Hétérozygote

$\mathrm{BALB} / \mathrm{cBy}$

Strie vasculaire et fibrocytes

[45]

Pcdh15 $5^{a v-3 j} \quad$ Protocadhérine 15

Hétérozygote

C57BL/6

Touffe ciliaire des cellules ciliées

Susceptibilité au bruit et au vieillissement cochléaire

\begin{tabular}{|c|c|c|c|c|c|}
\hline Cdh2 $23^{\text {ahl }}$ & Otocadhérine & Homozygote & C57BL/6, autres & Touffe ciliaire des cellules ciliées & [46] \\
\hline $\operatorname{Cdh} 23^{v}$ & Otocadhérine & Hétérozygote & $\mathrm{CBA} / ?$ & Touffe ciliaire des cellules ciliées & {$[47]$} \\
\hline Pmca2 KO & ATPase 2 & Hétérozygote & 129/BISw/C3H & Strie vasculaire et fibrocytes & {$[48]$} \\
\hline SODI KO & Superoxyde dismutase & Homozygote & $\begin{array}{l}129 / \mathrm{CD} 1 \\
\text { C57BL/6 }\end{array}$ & Mitochondrie & [49] \\
\hline TRPV4 KO & $\begin{array}{l}\text { Mechanically activated ion } \\
\text { channel }\end{array}$ & Homozygote & $\mathrm{C} 57 \mathrm{BL} / 6$ & Cellules ciliées et strie vasculaire & {$[50]$} \\
\hline
\end{tabular}

Tableau I. Gènes impliqués dans la susceptibilité de la cochlée au vieillissement et localisation dans l'organe de Corti. A. Photographie montrant une souris C57BL/6 d'un mois. B. Section transversale du tour basal de la cochlée d'une souris (microscopie photonique). L'organe de Corti (oC) est composé d'une cellule ciliée interne (CCI) et de trois cellules ciliées externes ( $C C E$ ), disposées de part et d'autre du tunnel de Corti ( $\mathrm{tC}$ ). L'oC est recouvert par la membrane tectoriale (MT) et il est séparé de la rampe tympanique (RT) par la membrane basilaire (mb). La lame spirale osseuse guide les fibres nerveuses qui relient les $\mathrm{CCl}$ aux corps cellulaires des neurones du ganglion spiral (GS). DC : Cellules de Deiters ; RV : rampe vestibulaire ; CC : canal cochléaire ; MR : membrane de Reissner ; LS : limbe spiral ; HC : cellules de Hensen ; fn : fibres du nerf auditif. Photographie : Marc Lenoir. Tableau : gènes impliqués dans la susceptibilité de la cochlée au vieillissement et à la combinaison bruit et vieillissement. 
dans le vieillissement de l'audition et de leurs interactions avec les facteurs environnementaux. Nous illustrons par quelques exemples comment les modèles de souris peuvent contribuer à la compréhension de la presbyacousie chez l'homme.

\section{Susceptibilité au bruit et vieillissement cochléaire} Henry en 1982 [14], et plus tard Li en 1992 [15], ont évalué la susceptibilité au bruit de différentes souches de souris. II a fallu attendre 1996 pour que Erway et ses collaborateurs montrent que la susceptibilité au bruit de la souche murine C57BL/6 obéissait à une transmission autosomique récessive, et postulent l'existence d'un locus ahl (age-related hearing loss) [16]. Dans une autre étude sur la souche $\mathrm{C} 57 \mathrm{BL} / 6$, les mêmes auteurs font le lien entre locus ahl et vieillissement cochléaire [17]. D'autres équipes localisent le locus sur le chromosome 10 [18], ce qui leur permet d'envisager un lien étroit entre la susceptibilité au bruit et le vieillissement cochléaire. À ce jour, trois nouveaux locus ont été associés à la vulnérabilité au bruit, et cinq autres sont impliqués à la fois dans la vulnérabilité au bruit et dans le vieillissement cochléaire (Tableau I).

\section{Presbyacousie sensorielle}

On sait depuis longtemps que certaines lignées de souris sont atteintes de surdités précoces [19]. L'implication d'une protéine constitutive des stéréocils des cellules sensorielles auditives a été mise en évidence à partir de la lignée B6/CAST-Cdh23 ${ }^{\text {CAST }}$, porteuse d'une mutation de la cadhérine 23. Dès l'âge d'un an, ces souris présentent une dégénérescence de l'organe de Corti suivant un gradient base-apex. D'autres lignées comme BALB/c, CBA/J, 129S6/SvEv montrent des altérations des cellules de soutien (noyau fragmenté, cytoplasme dense, etc.), alors que les cellules sensorielles sont intactes [20]. L'endommagement de ces cellules de soutien contribue aux pertes auditives liées à l'âge, de manière directe en affectant la micromécanique de la cochlée, ou indirectement en favorisant la perte des cellules sensorielles.

\section{Presbyacousie neurale}

Un modèle murin intéressant est la souris knock out $A C h R \beta 2$. Rappelons que les terminaisons des neurones ganglionnaires sont en contact avec les axones efférents dont les corps se situent dans l'olive supérieure latérale. Ces efférences latérales contiennent des neurotransmetteurs classiques (acétylcholine, dopamine, GABA) et des neuropeptides (enképhalines, dynorphines, calcitonin gene-related peptide [CGRP]) dont le rôle connu est de contrôler l'activité des neurones ganglionnaires et qui pourraient jouer un rôle neurotrophique important dans la survie de ces neurones. L'invalidation de la sous-unité $\beta 2$ des récepteurs nicotiniques de l'acétylcholine (AChR $\beta 2$ ) présents sur les terminaisons des neurones ganglionnaires entraîne une surdité et une diminution drastique du nombre de ces neurones. D'autre part, ainsi qu'on a pu le constater chez l'homme âgé [21], des phénomènes de regroupement de neurones survivants, trois ou quatre occupant la même gaine de myéline, ont été observés chez des souris présentant une dégénérescence neurale, telles que les souris C57BL/6 précédemment citées. Si le mécanisme à l'origine de ce phénomène est inconnu, le partage d'une même cellule de
Schwann pourrait promouvoir la survie des neurones en leur offrant une même cible synaptique.

En conclusion, le vieillissement cochléaire résulte de plusieurs atteintes. Les modèles murins permettent d'identifier les gènes et les mécanismes responsables de la presbyacousie, ce qui peut permettre de développer des traitements susceptibles de ralentir son évolution chez l'homme, voire de la stopper.

Le vieillissement neurosensoriel est-il inéluctable ? Une perspective de réparation peut-elle être envisagée ? Des travaux de plus en plus nombreux sur des îlots de cellules souches découvertes dans le système nerveux central pourraient permettre de répondre à ces questions.

\section{Vieillissement du cerveau et cellules souches adultes}

Dans les années 1960, plusieurs équipes démontraient la formation, chez le rongeur, de nouveaux neurones dans le cerveau adulte, au sein de régions particulières - dites niches -, notamment dans l'hippocampe et la zone sous ventriculaire (Figure 2). Ces travaux contredisaient le dogme d'un cerveau doté d'un nombre fixe de neurones déclinant avec l'âge et ouvraient ainsi la voie à l'étude de la neurogenèse adulte. Ainsi germait l'idée d'une neuropoïèse adulte jouant le rôle d'une «moelle » nerveuse qui, à l'image de la moelle osseuse pour le système hématopoiétique, régénèrerait le système nerveux au cours de la vie. II n'en est rien. Hormis dans ces niches, le renouvellement spontané de nos cellules nerveuses est probablement très faible ou inexistant. Des expériences basées sur l'étude de cerveaux humains ayant incorporé du carbone 14 pendant les essais atomiques ont démontré que nos neurones sont en majorité ceux que nous avons formés lors de notre développement embryonnaire et qu'ils ne sont pas remplacés au cours de la vie [22]. Il serait d'ailleurs difficile d'imaginer que ces cellules, support cellulaire de notre personnalité et stockant les informations accumulées au cours d'une vie entière, soient continuellement renouvelées comme le sont les cellules constitutives d'un épithélium. C'est le prix à payer pour avoir obtenu de l'évolution un système nerveux si performant.

Ces niches de cellules souches sont associées à la mémorisation et à l'apprentissage [23]. Le rôle de I'hippocampe dans la mémoire à long terme est bien connu et la réduction de la neurogenèse dans cette région provoque, chez les animaux, un déficit dans la mémoire spatiale et les apprentissages conditionnels. Chez l'homme, une relation entre la diminution des cellules souches de l'hippocampe et des déficits de la mémoire et de l'apprentissage semble exister [24]. 


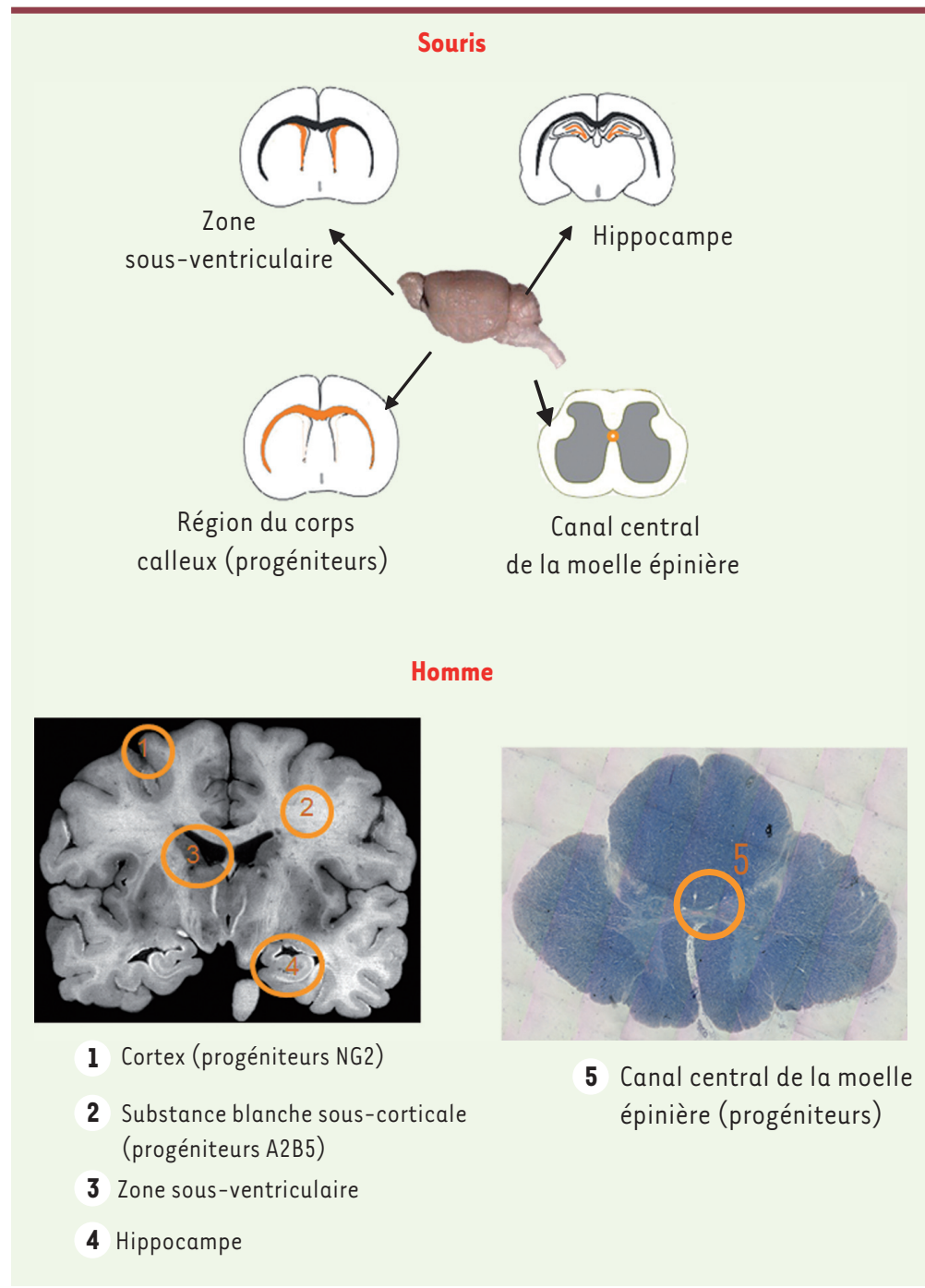

Figure 2. Localisation des principales régions (en orange) contenant des cellules souches et progénitrices neurales dans le cerveau de souris et humain (coupes coronales).

Ces niches ne sont pas épargnées par le vieillissement. Chez les rongeurs, la neurogenèse de l'hippocampe diminue d'un facteur 10 avec l'âge (entre deux mois et deux ans [25]) et s'accompagne d'un déclin de la mémorisation. On assiste aussi à une baisse de la neurogenèse dans la zone sous ventriculaire qui produit les jeunes neurones qui migrent dans le bulbe olfactif. Chez les primates âgés dont l'homme, la taille de l'hippocampe peut être diminuée et le nombre de jeunes neurones est dans ce cas réduit [26]. Plusieurs facteurs semblent concourir à cette baisse. L'augmentation du taux de corticostéroïdes avec l'âge pourrait y contribuer. En effet, il est connu que, lors d'un stress, le niveau élevé de corticostéroïdes influence négativement la neurogenèse [25]. Par ailleurs, ont été aussi incriminées : une modification de la structure de la niche affectant la vascularisation, l'entrée en sénescence des cellules souches, ou encore l'augmentation du taux de certaines cytokines comme le TGF $\beta$ I (transforming growth factor $\beta$ ) [25]. Cependant, la neurogenèse peut être réactivée chez les animaux âgés par l'exercice physique [25], comme on l'observe chez les jeunes animaux. Chez l'homme également, l'exercice apparaît bénéfique pour s'opposer à la réduction de l'hippocampe liée à l'âge et au déficit de mémorisation.

En dehors des niches, il existe des cellules dont le potentiel de prolifération et de différenciation est plus restreint que celui de cellules souches proprement dites, et que l'on désigne communément sous le terme de cellules progénitrices, exprimant NG2 (un protéoglycane à héparane sulfate) ou A2B5 (un ganglioside) (Figure 2). Ces cellules prolifèrent abondamment et jouent notamment le rôle de précurseurs d'oligodendrocytes, ces dernières assurant la myélinisation. Le vieillissement du cerveau humain s'accompagne d'une perte importante du nombre de fibres nerveuses myélinisées [27]. Les raisons de cette altération sont mal comprises : elle pourrait être due à une diminution du nombre de ces cellules progénitrices myélinisantes ou à l'altération de leurs propriétés .

En conclusion, parmi les multiples aspects du vieillissement du cerveau, l'atteinte des cellules souches au cours de ce processus constitue un élément relativement nouveau et dont l'étude est en pleine expansion. Le modèle rongeur, en raison de ses avantages, constitue un modèle intéressant pour explorer les relations cellules souches-vieillissement. Cependant, l'extrapolation au cerveau humain est hasardeuse, car ce dernier n'est pas seulement plus gros que celui des rongeurs, mais il est aussi bien plus complexe dans sa structure cellulaire et son organisation. Ainsi qu'on commence à l'observer chez les primates, il est probable qu'il existe d'autres niches de cellules souches dans le cerveau humain, comme par exemple l'amygdale [28]. D'où l'importance de développer des études chez l'humain, en dépit de toutes les difficultés que cela comporte, afin de préserver et utiliser pleinement notre capital « cellules souches neurales » et lutter ainsi contre le vieillissement.

\section{Microcebus murinus, un modèle primate pour l'étude du vieillissement cérébral et des maladies neurodégénératives associées}

\section{Pourquoi un modèle primate lémurien ?}

Avec l'accroissement de la longévité, le nombre de personnes atteintes de maladie d'Alzheimer augmente considérablement dans nos sociétés. En effet, l'âge constitue le facteur de risque le plus important de 


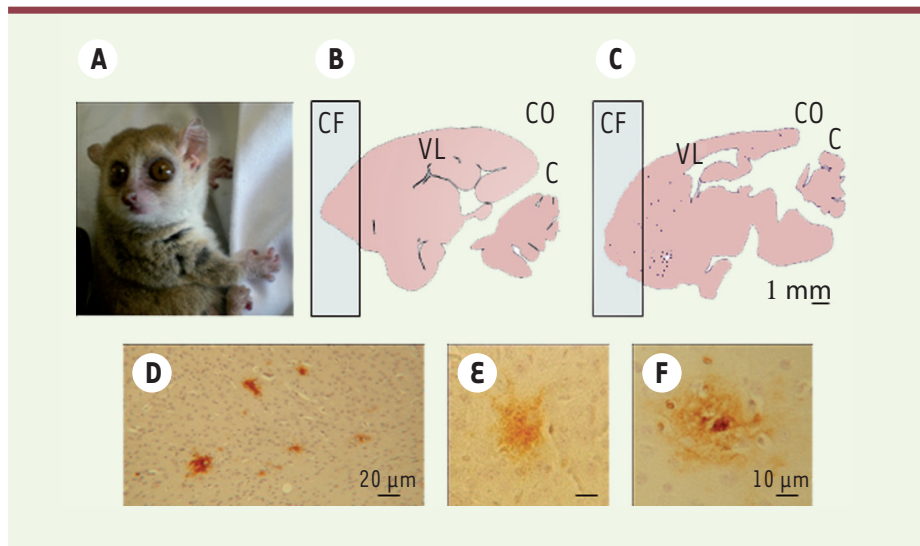

Figure 3. Analyse histologique des cerveaux de microcèbes. A. Photographie montrant un microcèbe adulte, un des plus petits primates lémuriens existant. $B$ - $C$. Coupes histologiques sagittales de cerveau ( $\boldsymbol{B}$, un lémurien adulte ; $\boldsymbol{C}$, un lémurien âgé présentant des plaques amylö̈des). CF : cortex frontal ; $C O$ : cortex occipital ; C : cervelet ; VL : ventricule latéral gauche. D-F. Photographies de microscopie optique montrant la présence de plaques amyloïdes dans le cortex frontal par marquage immunocytochimique.

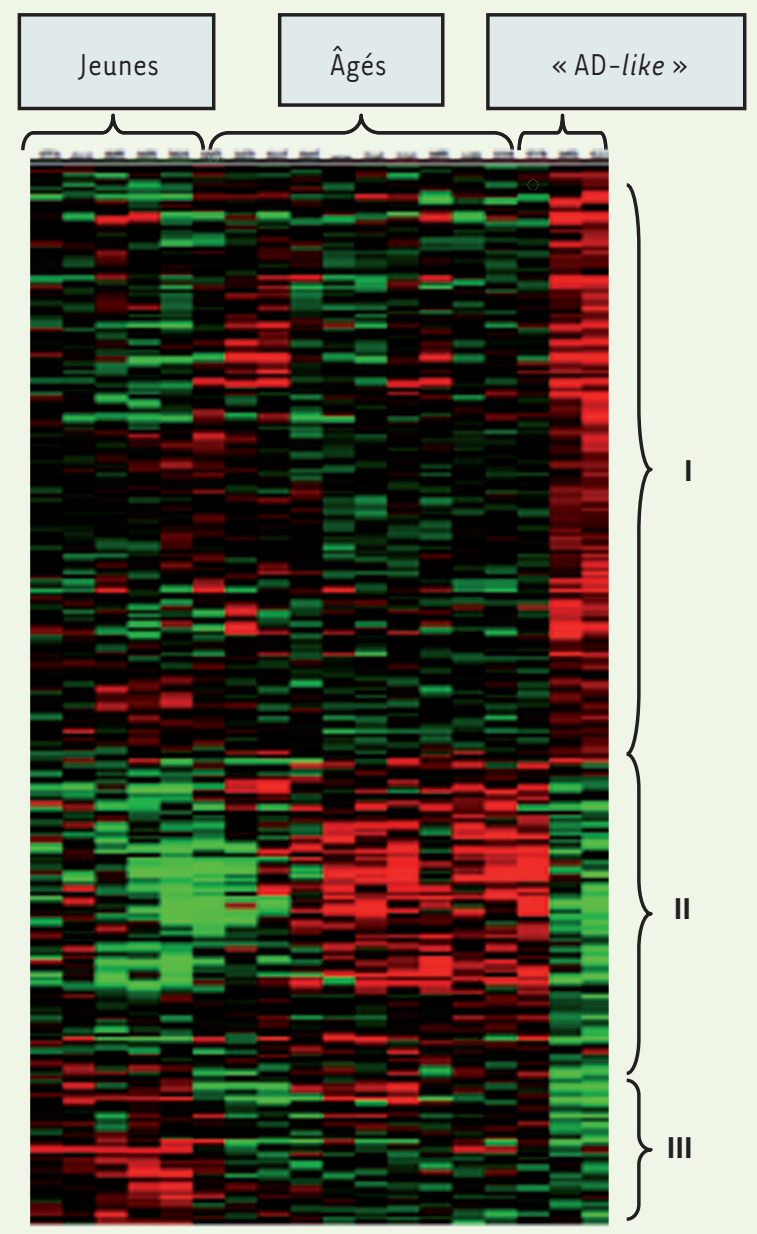

survenue de maladie d'Alzheimer. Cette pathologie est complexe, multifactorielle et encore mal connue. II est par conséquent important de mieux comprendre les mécanismes moléculaires qui la sous-tendent et qui la différencient d'un vieillissement physiologique normal. Pour cela, les modèles animaux sont indispensables. Les souris sauvages ne présentent pas spontanément dans leur cortex d'agrégats protéiques caractéristiques de la maladie d'Alzheimer, tels que les plaques amyloïdes et/ou les dégénérescences neurofibrillaires, alors que ces agrégats sont retrouvés naturellement dans le cortex des primates. De plus, les fonctions cognitives et les comportements des rongeurs n'atteignent pas le même degré de sophistication que ceux des primates. Chez ces derniers, la croissance des aires corticales frontales et temporales, indispensables aux processus mnésiques et aux apprentissages, a permis un développement des fonctions cognitives hautement intégratives. Par ailleurs, des études transcriptomiques réalisées sur des échantillons de cerveaux d'individus âgés ont montré que les changements d'expression de gènes sont mieux conservés entre primates qu'entre primates et souris [29]. Cependant, l'utilisation des primates pose de nombreux problèmes éthiques, de coût, de longévité et de cohortes. Nous avons choisi d'étudier un autre modèle primate, Microcebus murinus, un petit primate lémurien originaire de Madagascar (Figure 3A), pour étudier le vieillissement cérébral et les maladies neurodégénératives qui lui sont associées (Figure 3B et 3C).

\section{Une large palette d'études possibles:} du génome au comportement

Le microcèbe murin, Microcebus murinus, a une espérance de vie de quatre à six ans en milieu naturel qui peut atteindre 10 voire 13 ans en captivité. Les animaux

Figure 4. Profils transcriptionnels du cortex temporal de 18 microcèbes. Les cerveaux de six jeunes microcèbes adultes, dix microcèbes âgés sains et deux porteurs de plaques amyloïdes ont été analysés. Après l'analyse statistique des données transcriptomiques, la classification hiérarchique des gènes différentiellement exprimés montre un profil spécifique pour chacun des trois groupes de microcèbes. Ce profil se divise en trois régions distinctes : I - forte surexpression des transcrits chez les animaux ayant une pathologie Alzheimer-like; II - transcrits sous exprimés chez les animaux Alzheimer-like mais surexprimés chez les âgés sains ; III - transcrits sous-exprimés chez tous les microcèbes âgés, sains et malades, par rapport aux jeunes adultes. Code couleur : en rouge, les gènes surexprimés; en vert, les gènes sous exprimés; en noir, pas de changement d'expression. 
de plus de 5 ans ont de nombreuses similitudes avec les humains âgés [30]. Par exemple, environ $5 \%$ des microcèbes présentent, principalement au niveau du cortex, des plaques amyloïdes denses (Figure 3C) [31], une atrophie corticale et une dilatation ventriculaire, visibles en IRM et plus importantes que celles qui sont observées au cours du vieillissement physiologique [32]. Les animaux porteurs de plaques amyloïdes sont appelés Alzheimer disease-like (Figure 3C) par opposition aux adultes normaux (Figure $3 B$ ).

Au niveau comportemental, bien que curieux, les microcèbes sont très émotifs et facilement stressés. C'est pour cela que, depuis plusieurs années, l'évaluation de leurs capacités mnésiques reste difficile et que les tests ont dû être adaptés à leur personnalité [33]. Cependant, les différents aspects de la mémoire, mémoire de travail, mémoire spatiale ou encore mémoire à long à terme, peuvent être évalués. Microcebus murinus a un odorat très sensible que nous utilisons pour tester ses capacités mnésiques. Nous avons montré, en utilisant cette modalité olfactive via des approches de type go/no go, que des femelles très âgées (13 ans) pouvaient conserver des capacités cognitives intactes, suggérant que l'âge n'est pas systématiquement associé à des déficits cognitifs [34]. Par une approche transcriptomique (puces Affymetrix HG 133plus2), nous avons comparé les transcrits du cortex temporal droit de microcèbes âgés sains avec ceux de sujets jeunes adultes et de sujets ayant une maladie Alzheimer-like [35]. Les profils d'expression génique observés sont spécifiques de l'âge ou de la pathologie des animaux (Figure 4). Parmi les gènes dont l'expression est modifiée, 47 discriminent de façon très efficace le vieillissement de la pathologie neurodégénérative. Ces gènes sont impliqués dans la plasticité synaptique, la synthèse des protéines et dans des mécanismes de régulation de la transcription. Ces résultats suggèrent qu'au cours du vieillissement physiologique, la surexpression des gènes impliqués dans la synthèse protéique pourrait participer à des processus de compensation, alors que celle-ci serait abolie chez les animaux ayant une pathologie Alzheimer-like.

En conclusion, ce petit lémurien est un modèle très pertinent, non seulement pour décrypter les mécanismes moléculaires impliqués dans le vieillissement cérébral et les maladies neurodégénératives associées, mais aussi pour analyser ses comportements cognitifs et sociaux, qui se rapprochent beaucoup plus de ceux de l'homme que de ceux des rongeurs habituellement utilisés.

Dans le futur, une meilleure compréhension des mécanismes concourant à minimiser les effets du vieillissement, passera nécessairement par un effort d'intégration des nombreuses données issues de la diversité de ces organismes modèles. $\diamond$

\section{SUMMARY}

The diversity of aging models

Most of the signalling pathways involved in aging regulation have been recently found well conserved at various levels throughout the evolution. Taking this into account, a diversity of model organisms, including worms, rodents, and lemurs as well, allows to address different questions: how to understand the interactions between genetic and environmental factors while challenging theories of aging, to preserve hearing integrity, to fight against senescence of neural stem cells, or to explore brain fitness from gene expression to cognitive and social behavior? Here are the main issues that can be considered, stressing the complementarities of the models. The differentiation of aging physiological aspects from those induced by age-related pathologies will also be specified. By emphasizing recent ability of technologies to promote new aging insights, we discuss towards a better understanding of mechanisms governing aging. $\diamond$

\section{CONFLIT D'INTÉRÊTS}

Les auteurs déclarent n'avoir aucun conflit d'intérêts concernant les données publiées dans cet article.

\section{RÉFÉRENCES}

1. The $C$. elegans Sequencing Consortium. Genome sequence of the nematode $C$. elegans: a platform for investigating biology. Science 1998 ; 282 : 2012-18.

2. Johnson TE, Wood WB. Genetic analysis of life-span in Caenorhabditis elegans. Proc Natl Acad Sci USA $1982 ; 79$ : 6603-7.

3. Kenyon C, Chang J, Gensch $\varepsilon$, et al. A C. elegans mutant that lives twice as long as wild type. Nature $1993 ; 366: 461-4$.

4. Dong MQ, Venable J D, Au N, et al. Quantitative mass spectrometry identifies insulin signaling targets in C. elegans. Science $2007 ; 317: 660-3$.

5. Van Raamsdonk JM, Hekimi S. Deletion of the mitochondrial superoxide dismutase sod-2 extends lifespan in Caenorhabditis elegans. PLoS Genet $2009 ; 5$ : el000361.

6. Yang W, Hekimi S. A mitochondrial superoxide signal triggers increased longevity in Caenorhabditis elegans. PLoS Biol $2010 ; 8$ : el000556.

7. http://ibg.colorado.edu/tj-lab/worms/gerontogenes/worm_gerontogenes. html

8. Château MT, Araiz C, Descamps S, Galas S. Klotho interferes with a novel FGF-signalling pathway and insulin/lgf-like signalling to improve longevity and stress resistance in Caenorhabditis elegans. Aging $2010 ; 2: 567-81$.

9. Arking DE, Krebsova A, Macek M Sr, et al. Association of human aging with a functional variant of klotho. Proc Natl Acad Sci USA 2002 ; 99 : 856-61.

10. Araiz C, Château MT, Descamps S, Galas S. Quantitative genomics in Caenorhabditis elegans: Identification strategies for new human therapeutic targets and molecular mechanisms. ITBM-RBM 2008 ; 29 : 289-96.

11. Willott JF, Hnath Chisolm T, Lister JJ. Modulation of presbycusis: current status and future directions. Audiol Neurootol $2001 ; 6$ : 231-49.

12. Schuknecht HF, Gacek MR. Cochlear pathology in presbycusis. Ann Otol Rhinol Laryngol 1993; $102: 1-16$.

13. Menardo J, Tang $Y$, Ladrech $S$, et al. Oxidative stress, inflammation and autophagic stress as the key mechanisms of premature age-related hearing loss in SAMP8 mouse cochlea. Antioxid Redox Signal $2012 ; 16$ : 263-74.

14. Henry KR. Influence of genotype and age on noise-induced auditory losses. Behav Genet $1982 ; 12: 563-73$.

15. Li HS. Influence of genotype and age on acute acoustic trauma and recovery in CBA/Ca and C57BL/6) mice. Acta Oto-Laryngol 1992; 112 : 956-67.

16. Erway LC, Shiau YW, Davis RR, Krieg EF. Genetics of age-related hearing loss in mice. III. Susceptibility of inbred and Fl hybrid strains to noise-induced hearing loss. Hear Res $1996 ; 93: 181-7$.

17. Davis RR, Newlander JK, Ling X-B, et al. Genetic basis for susceptibility to noise-induced hearing loss in mice. Hear Res $2001 ; 155: 82-90$.

18. Johnson KR, Erway LC, Cook SA, et al. A major gene affecting age-related hearing loss in C57BL/ 6J mice. Hear Res 1997 ; $114: 83-92$.

19. Kocher W. Investigations of the genetics and pathology of the development of a late-appearing hereditary type of deafness in the mouse. Arch OhrenNasen Kehlkopfheilkd $1960 ; 177$ : 108.

20. Ohlemiller KK, Gagnon PM. Cellular correlates of progressive hearing loss in 129S6/SvEv mice. J Comp Neurol 2004 ; 469 : 377-90.

21. Felder $\varepsilon$, Kanonier $G$, Scholtz A, et al. Quantitative evaluation of cochlear neurons and computer-aided three-dimensional reconstruction of spiral ganglion cells in humans with a peripheral loss of nerve fibers. Hear Res 1997 ; $105: 183-90$. 


\section{RÉFÉRENCES}

22. Spalding KL, Bhardwaj RD, Buchholz BA, et al. Retrospective birth dating of cells in humans. Cell $2005 ; 122: 133-43$.

23. Shors TJ, Miesegaes G, Beylin A, et al. Neurogenesis in the adult is involved in the formation of trace memories. Nature $2001 ; 410: 372-6$.

24. Coras R, Siebzehnrubl FA, Pauli $\varepsilon$, et al. Low proliferation and differentiation capacities of adult hippocampal stem cells correlate with memory dysfunction in humans. Brain $2010 ; 133$ : 3359-72.

25. Jessberger S, Gage FH. Stem-cell-associated structural and functional plasticity in the aging hippocampus. Psychol Aging $2008 ; 23: 684-91$.

26. Knoth $R$, Singec $I$, Ditter $M$, et al. Murine features of neurogenesis in the human hippocampus across the lifespan from 0 to 100 years. PLoS One $2010 ; 5$ : e8809.

27. Marner L, Nyengaard JR, Tang $Y$, Pakkenberg B. Marked loss of myelinated nerve fibers in the human brain with age. J Comp Neurol 2003; 462: 144-52.

28. Bernier PJ, Bedard A, Vinet J, et al. Newly generated neurons in the amygdala and adjoining cortex of adult primates. Proc Natl Acad Sci USA $2002 ; 99$ : 11464-9.

29. Loerch PM, Lu T, Dakin KA, et al. Evolution of the aging brain transcriptome and synaptic regulation. PLoS One $2008 ; 3$ : e3329.

30. Perret M, Aujart F. Vieillissement et rythmes biologiques chez les primates. Med Sci (Paris) 2006 ; $22: 279-83$.

31. Mestre-Frances N, Keller $\varepsilon$, Calenda A, et al. Immunohistochemical analysis of cerebral cortica and vascular lesions in the primate Microcebus murinus reveal distinct amyloid betal-42 and betal-40 immunoreactivity profiles. Neurobiol Dis $2000 ; 7: 1-8$.

32. Kraska A, Dorieux 0 , Picq J-L, et al. Age-associated cerebral atrophy in mouse lemur primates. Neurobiol Aging $2011 ; 32: 894-906$.

33. Trouche SG, Maurice T, Rouland S, et al. The three panel runway maze adapted to Microcebus murinus reveals age-related differences in memory and perseverance performances. Neurobio Learn Mem $2010 ; 94: 100-6$.

34. Joly M, Deputte B, Verdier JM. Age effect on olfactory discrimination in a non-human primate, Microcebus murinus. Neurobiol Aging $2006 ; 27$ : 1045-9.

35. Abdel Rassoul R, Alves S, Pantesco V, et al. Distinct transcriptome expression of the temporal cortex of the primate Microcebus murinus during brain aging versus Alzheimer's disease-like pathology. Plos One 2010 ; 5 : el22770.

36. Guo Y, Zhang C, Du X, et al. Morphological and functional alterations of the cochlea in apolipoprotein $\varepsilon$ gene deficient mice. Hear Res $2005 ; 208: 54-67$.

37. Johnson KR, Zheng $\mathrm{QY}$. AhI2, a second locus affecting age-related hearing loss in mice. Genomics $2002 ; 80: 461-4$.

38. Nemoto M, Morita $Y$, Mishima $Y$, et al.Ahl3, a third locus on mouse chromosome 17 affecting agerelated hearing loss. Biochem Biophys Res Commun $2004 ; 324$ : 1283-8.
39. Minowa 0, Ikeda K, Sugitani $y$, et al., Altered cochlear fibrocytes in a mouse model of DFN3 nonsyndromic deafness. Science $1999 ; 285$ : 1408-11.

40. Hildebrand MS, de Silva MG, Klockars T, et al. Characterisation of DRASIC in the mouse inner ear. Hear Res $2004 ; 190: 149-60$.

41. Kujoth GC, Hiona A, Pugh TD, et al., Mitochondrial DNA mutations, oxidative stress, and apoptosis in mammalian aging. Science $2005 ; 309: 481-4$.

42. Bao J, Lei D, Du Y, et al. Requirement of nicotinic acetylcholine receptor subunit beta2 in the maintenance of spiral ganglion neurons during aging. J Neurosci $2005 ; 25$ : 3041-5.

43. Johnson KR, Zheng QY, Bykhovskaya Y, et al. A nuclear-mitochondrial DNA interaction affecting hearing impairment in mice. Nat Genet $2001 ; 27$ : 191-4.

44. Noben-Trauth K, Zheng QY, Johnson KR, et al., mdfw: a deafness susceptibility locus that interacts with deaf waddler (dfw). Genomics 1997 $44: 266-72$.

45. Zheng $Q Y$, Yan D, Ouyang XM, et al. Digenic inheritance of deafness caused by mutations in genes encoding cadherin 23 and protocadherin 15 in mice and humans. Hum Mol Genet 2005 ; 14 : 103-11.

46. Davis RR, Newlander JK, Ling $X$, et al. Genetic basis for susceptibility to noise-induced hearing loss in mice. Hear Res 2001 ; 155 : 82-90.

47. Holme RH, Steel KP. Stereocilia defects in waltzer (Cdh23), shakerl (Myo7a) and double waltzer/shakerl mutant mice. Hear Res $2002 ; 169: 13-23$.

48. Davis RR, Kozel $P$, Erway LC. Genetic influences in individual susceptibility to noise: a review. Noise Health $2003 ; 5: 19-28$.

49. Keithley $\varepsilon M$, Canto $C$, Zheng $Q Y$, et al., $\mathrm{Cu} / \mathrm{Zn}$ superoxide dismutase and agerelated hearing loss. Hear Res $2005 ; 209$ : 76-85.

50. Tabuchi K, Suzuki M, Mizuno A, et al. Hearing impairment in TRPV4 knockout mice. Neurosci Lett 2005 ; 382 : 304-8.

51. Terret C, Solari F. L'homéostasie métabolique au cœur du vieillissement. Med Sci (Paris) $2012 ; 28: 311-5$.

52. Brunet $A$. Bien vieillir : la voie de signalisation insuline-FOXO et la longévité. Med Sci (Paris) $2012 ; 28: 316-20$.

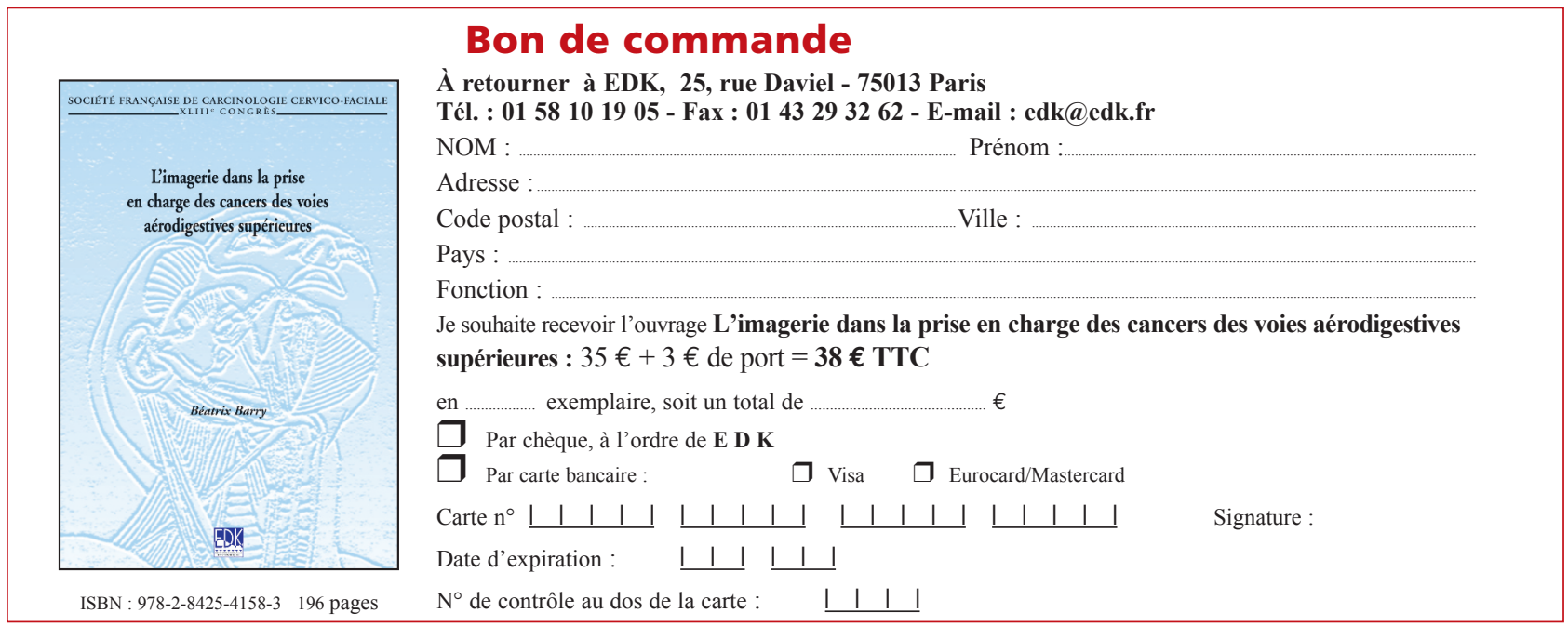

TIRÉS À PART

S. Galas 